\title{
Students' use of symbolic forms when constructing equations of boundary conditions
}

\author{
Qing X. Ryan ${ }^{1}$ and Benjamin P. Schermerhorn ${ }^{1,2}$ \\ ${ }^{1}$ Department of Physics \& Astronomy, California State Polytechnic University Pomona, \\ Pomona, California 91768, USA \\ ${ }^{2}$ Department of Physics, California State University, Fullerton, Fullerton, California 92831, USA
}

(Received 2 January 2020; accepted 3 March 2020; published 24 April 2020)

\begin{abstract}
In an effort to examine students' understanding about the structure of boundary conditions in upperdivision courses, think-aloud interviews were conducted in the context of both quantum mechanics and electricity and magnetism. In the quantum mechanics task, students were presented with a standard potential step scenario. In the electricity and magnetism task, students were given a classical question about electromagnetic waves at the boundary of two media. The analysis of the interviews was guided by the use of a symbolic forms perspective. In this paper, we report the symbolic forms identified in students' work and provide examples of students' reasoning. Generally speaking, students were able to generate the correct symbolic templates while having difficulties with the underlying conceptual ideas. We also discuss instructional implications from this study.
\end{abstract}

DOI: 10.1103/PhysRevPhysEducRes.16.010122

\section{INTRODUCTION}

Investigation aimed at understanding students' use of mathematics in physics is a major research interest at both the introductory and upper-division level [1]. A growing body of research at the upper-division level suggests that students continue to struggle with physics concepts and mathematical tools in advanced physics topics (see, e.g., Refs. [2,3]). Using mathematics to solve physics problems is an important skill and it is often listed as an explicit goal for upper-division courses. [2,4].

There are two common approaches in understanding the difficulties students encounter when using mathematics in physics. The first approach is to characterize physicists' use of mathematics which can help produce instructional tools to help students to be more expertlike. The second approach is to describe students' behavior and how students make sense of and use mathematics to solve physics problems. One example of the latter approach can be represented by Sherin's work on students' use of symbolic forms [5] (discussed more in Sec. II). However, Sherin [5] acknowledges that these approaches can be connected by exploring students' naive physics understanding, which provides an important part of the conceptual basis in terms of which equations are understood. The symbolic form framework is connected to other earlier researches on naive

Published by the American Physical Society under the terms of the Creative Commons Attribution 4.0 International license. Further distribution of this work must maintain attribution to the author(s) and the published article's title, journal citation, and DOI. physics such as the "knowledge-in-pieces" [6] and "resources" [7] framework. We are looking for cognitive resources that students have, so that we can help students be more productive by bridging their reasoning and expert thinking rather than getting rid of these naive ideas.

In Sherin's work [5], he discovered that students' understand physics equations in terms of a vocabulary of elements that he called symbolic forms. He found out that students have this intuition of what the equation should express in a fundamental sense. For example, students construct an equation in the format of "something equal to something else" and would write an equals sign between two terms as $\square=\square$. Students have a basic and generic intuition of what this equation says: two amounts, each associated with a side, are the same. It was argued that equations can be understood in terms of generic intuitions that cut across expert domains [5] and it has been demonstrated that students often construct physics equations using these intuitions.

We believe that if the ultimate goal of instruction is to help students to become more expertlike, we need to understand how students currently interpret and work with equations. Since the use of symbolic form was manifested in multiple physics contexts as demonstrated by Sherin, it is productive to study students' use of these symbolic forms because the proper use of these forms can help or hinder them in more than one context. Furthermore, we would like to see if upperdivision students are continuing to use symbolic forms when constructing physics equations and whether that can be more or less productive for them in different contexts.

In order to gain a deeper understanding of how equation construction can be the same or different depending on 
various physics concepts, we would like to investigate a topic that appears multiple times across the advanced undergraduate physics curriculum [8]. One such topic is boundary conditions, which refer to the conditions physical quantities must satisfy at the boundary between two regions. Boundary conditions are used in a variety of contexts, including classical mechanics, quantum mechanics (QM), and throughout electricity and magnetism $(\mathrm{E} \& \mathrm{M})$. For example, in $\mathrm{E} \& \mathrm{M}$, when there is no free charge or current at the boundary, boundary conditions are

$$
\begin{aligned}
\vec{E}_{1}^{\|} & =\vec{E}_{2}^{\|}, \\
\epsilon_{1} \vec{E}_{1}^{\perp} & =\epsilon_{1} \vec{E}_{2}^{\perp}, \\
\vec{B}_{1}^{\perp} & =\vec{B}_{2}^{\perp}, \\
\vec{B}_{1}^{\|} / \mu_{1} & =\vec{B}_{2}^{\|} / \mu_{2} .
\end{aligned}
$$

In this study, we investigate how students construct the equations of boundary conditions in two different contexts: quantum mechanics and electricity and magnetism. Thinkaloud interviews were conducted and symbolic forms in students' work were identified. We present common symbolic forms in both contexts, as well as some examples of student reasoning. We also compare symbolic form with an alternative model (principle-based schemata view) for investigating student reasoning in Sec. V. Limitations of this study and pedagogical implications are also discussed.

\section{LITERATURE REVIEW}

\section{A. Overview of symbolic forms}

The symbolic forms framework was originally established by Sherin [5] after observations that physics students would often construct equations from "a sense of what they wanted to express." In order to describe students' understanding of the mathematical representation involved in equation construction, a symbolic form is composed of two components. The first of these components is the symbol template, which represents the structural aspects of equations. For example, both an equation for finding total energy from kinetic and potential energies $\left(E_{\mathrm{tot}}=K+U\right)$ and the equation for finding total momentum of a two object system ( $p_{\text {syst }}=p_{a}+p_{b}$ ) have the same template, $\square+\square$. The second component of a symbolic form is the conceptual schema which is the acontextual mathematical justification for the template. The conceptual schema associated with the $\square+\square+\cdots$ template is "amounts of a generic substance, associated with terms, that contribute to a whole" [5]. Together these components are the parts-of-a-whole symbolic form.

Equations in physics are often much more complex and as such can take on a nested structure. For example, students reasoning about an air resistance task constructed the equation $a(t)=-g+[f(v)] / m$. Students' inclusion of the negative sign and specific language referring to the opposition of the terms on the right-hand side of the equation leads Sherin to identify an overall opposition symbolic form, $\square-\square$, which represents "two terms, separated by a minus sign, associated with influences that work against each other." Additionally, one of the terms could be seen as including both the prop+ and propsymbolic forms which indicate proportionality and inverse proportionality, with the corresponding symbolic template being $(\ldots \square \ldots) / \ldots$ and $\ldots /(\ldots \square \ldots)$, respectively. The identification of these symbolic forms is inherent on the way students describe the associated underlying mathematics that is necessary to construct the equation.

Since symbolic forms only refer to the mathematical symbols, Sherin states that students can still use symbolic forms properly (i.e., the equation correctly expresses their intuition) even if the underlying physics is not correct [5]. In one example, students write an equation for a coefficient of friction as a sum of two components, one constant and another that is inversely proportional to the mass of the object. While students correctly express inverse proportionality as part of the equation, $\mu=\mu_{1}+C \mu_{2} / m$, the physics of the equation is not true.

Furthermore, the use of symbolic forms extends to analyzing students' interpretation of equations. Students with understanding of mathematical relationships used in physics equations can identify the structures of an equation and read out the associated information.

\section{B. Previous use of symbolic forms}

Symbolic forms has recently been used more widely in physics education research, as well as expanded to education in both mathematics and chemistry disciplines. In the introductory electrostatics context, the conceptual schema of symbolic forms were adapted to explain what cued students toward integration in electrostatics problems [9]. They found students discussing integration in terms of the underlying mathematical ideas of parts of a whole and dependence. Parts of a whole was used by students who acknowledged the need to sum up the effects of small charges along a charged rod. The dependence symbolic form, signified by an expression that "depends on" a given variable or quantity (template $[\ldots x \ldots]$ ), was invoked by students attending to position along the rod and then including the variable in the expression.

Kuo et al. addressed introductory students qualitative reasoning and "processing" of the kinematics equation, $v_{f}=v_{i}+a t$ [10]. Kuo et al. compare case studies of students either interpreting or failing to interpret the base + change symbolic form with template $(\square+\Delta)$ and conceptual schema "one is the base value; the other is a change to that base" [5]. The student who does not invoke the base + change symbolic form reasons formulaically while the other interprets the mathematical structure in terms of the physical situation. 
Expanding symbolic forms to analyze student equation construction in upper-division physics, Schermerhorn and Thompson identified and defined new symbolic forms to address students constructing differential length vectors in non-Cartesian coordinate systems [11]. Researchers found that students had a sense of the template and structures that were needed to write the equation but struggled with the conceptual ideas related to what terms needed to be expressed. In upper-division quantum mechanics, Dreyfus et al. [12] theorize transformation and eigenvalue-eigenvector symbolic forms for quantum mechanics in an effort to develop theory around mathematical sense making. Using these symbolic forms they discuss interpretations of student understanding of the eigenvalue equation.

Symbolic forms has been used beyond physics education research by both the chemistry and mathematics education research communities. Jones [13] defined several symbolic forms for students' different conceptions of definite integrals. Accounting for the different conceptualizations of integration (e.g., area and perimeter, Riemann sum, and function matching), many of the identified forms had identical templates with differing conceptual schema. Further discussion of this work in light of a conceptual blending approach to students' symbolic forms use [14] envisions these as a single symbolic form blended with different contextual interpretations of the integral.

Becker and Towns [15] extended symbolic forms to analysis of physical chemistry students' use of partial derivatives in thermodynamics. In several cases, recall of processes, like taking a total derivative, or concepts, such as the derivative being zero for constants, mediated students' construction of and reasoning with expressions in upperdivision contexts.

\section{METHODOLOGY}

In this study, we investigate students' understanding of equation construction in the context of boundary conditions. Two questions involving the use of boundary conditions were constructed (Fig. 3), one in QM and the other in E\&M. Both questions involved common textbook scenarios that students often encounter in class. In the quantum mechanics task, students were presented with a standard potential step. In the electricity and magnetism task, students were given a classical question about electromagnetic waves at the boundary of two media.

Fourteen individual think-aloud interviews were conducted. At the beginning of the interview, the interviewer explained to the students what to expect: "This is a thinkaloud interview where you verbalize your thinking while working through the question. I will try not to interrupt and let you do most of the talking. I know thinking-aloud probably does not come naturally to many people, so if you forget to talk, I might remind you to tell me what you are thinking. Or I might ask clarifying questions. Don't be alarmed if I ask you a question, it doesn't mean you did something wrong, it is simply because I am interested in your reasoning." Students then began the interview process. When the interviewer interacted with the students with questions, the dialog followed a Piaget style clinical interview method [16]. All students interviewed were undergraduate physics majors.

The QM question was provided to eight students at $\mathrm{Cal}$ Poly Pomona (CPP) who were enrolled in the year-long quantum mechanics course (four interviews were conducted during the first quarter and the rest were conducted during the second quarter). The topic of potential well or step was covered in class during the first few weeks in the first quarter. The E\&M question was given to two CPP students and 6 other students at University of Colorado Boulder. All eight students were interviewed after they had taken a year of E\&M. The two CPP students belong to the same group of students who also did the QM question: the E\&M question was given first and the QM question was given later during the same interview session. Interviews were first transcribed and then analyzed for common patterns in the ideas used by students when constructing boundary conditions. Further analysis mapped these common ideas onto symbolic forms, focusing on what kind of conceptual understanding was associated with a particular symbolic template.

In the interview questions for quantum mechanics, the interviewer started out with the question shown in Fig. 1. If students struggled significantly with the first question, the interviewer presented students with a scaffolding question (Fig. 2). Note that this question is exactly the same as the original question but with the mathematical expression of the waves explicitly given. No scaffolding questions were given to students doing the E\&M problem. Six of the eight students were all able to construct some kind of equations in the first set of the E\&M interviews. The remaining two interviews on the $\mathrm{E} \& \mathrm{M}$ problem were conducted with students who were also given the QM problem. Because of time constraints we had to move on to the QM part of the interview after the E\&M part, so there was no time allowed for the scaffolding question.

\section{RESULTS}

\section{A. General flow of symbolic forms}

Despite the differences in the physics context, these two types of problems are similar in the structure of how equations are formed. Given the similarity of the symbolic forms used between the two scenarios, we outlined the general flow of equation structure that were observed from students' work, using symbolic forms in one graph (Fig. 3).

Because of the increased complexity of the upperdivision content, we drew the general flow in a hierarchy format for organizational purposes. We do not indicate that students always follow such a linear process. For example, the "same amount" symbolic form outlined in the flow 


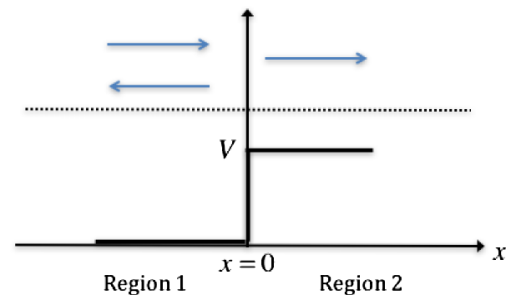

(a)

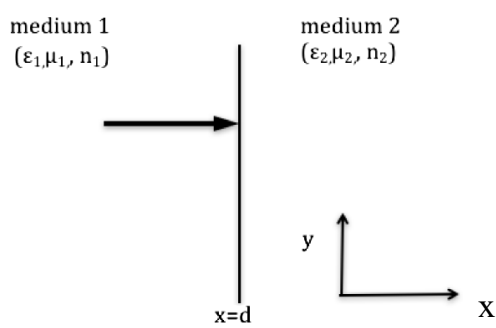

(b)

FIG. 1. (a) QM interview question: potential step scenario in quantum mechanics. Boundary conditions used in this scenario are $\psi_{1}=\psi_{2}$ and $d \psi_{1} / d x=d \psi_{2} / d x$ at $x=0$. There is a potential step $V$ with walls at $x=0$. Consider a particle with energy $E>V$ incident from the left. Question 1: In quantummechanics we use probability waves to help us find the probability of finding a particle within a certain region. Find a relationship between the amplitudes of the incoming, reflected and transmitted waves. (b) E\&M interview question: electromagnetic waves at the boundary of two media. Boundary conditions used in this scenario are $E_{1}^{\|}=E_{2}^{\|}$at $x=d$. Consider a plane electromagnetic wave of frequency $\omega$, polarized in the $y$-direction, approaches a boundary (located at $x=d$ ) between two linear media fromthe left at normal incidence, as schematically shown in Fig. 1(b). Apply appropriate boundary conditions for the $E$ field to write down a mathematical expression that relates the amplitudes of the electric fields of the incident, reflected and transmitted waves.

chart can be thought of as a first-order form students came up with. Sometimes several subsymbolic forms are used to further span the equation: parts of a whole, whole-part, and opposition. Some students stopped at the same amount form without further spanning one side of the equation into other terms. At this point they were given a scaffolding question asking as shown in Fig. 2. After this question, all students were able to continue and eventually all students used additional symbolic forms other than the "same amount." Below we discuss each symbolic form and provide some examples of students' work to connect the symbolic form to the physics context. When quoting transcripts from interviews, we use $\mathrm{S}$ for student and I for interviewer.

\section{B. Symbolic forms}

The scaffolding question in quantum mechanics was provided to five (S3, S4, S5, S7, S8) students. Overall, we

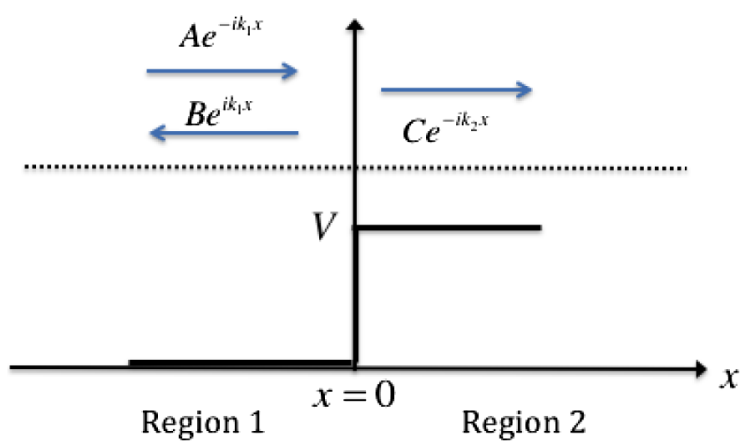

FIG. 2. Backup scaffolding question for quantum mechanics. There is a potential step $V$ with walls at $x=0$. Consider a particle with energy $E>V$ incident from the left. Question 1: In quantum mechanics we use probability waves to help us find the probability of finding a particle within a certain region. Find a relationship between $A, B$ and $C$. found similar patterns in the use of symbolic forms between QM and E\&M (Table I). In this table, we listed the number of students using each symbolic form and then we will discuss each form and provide examples of students' work.

\section{Same amount}

The symbolic template for this form is $\square=\square$. The description of this symbolic form is as follows (as defined by Sherin [5]): two amounts, each associated with a side, are the same. It is a symbol template for an equation in which two expressions are set equal. These two expressions can be of any sort, since symbolic forms can be nested within each other (i.e., the $\square$ can be filled in with any expression). We found out all 16 students in both QM and E\&M interviews used this symbolic form when constructing the equation: they either wrote down an equation with some quantity equating to some other quantity (or combinations of other quantity), or said in words that something must equal to something else, or explicitly drew a symbolic template in their work, even though they did not know what goes into that template. Student S3 uses this reasoning in the quantum mechanics context.

S3: "Ok. One thing that I am thinking about is whether or not [... inaudible] infinite square well. This is a dimension, right? This is some barrier. So I am thinking of the well. If it is passing through this way... So some wave is

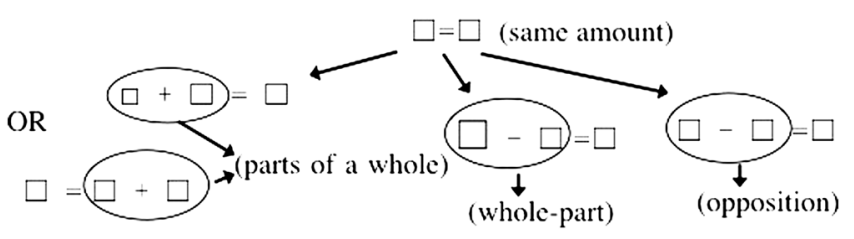

FIG. 3. General flow of students' use of symbolic forms. 
TABLE I. Number of students using each symbolic form.

\begin{tabular}{|c|c|c|c|c|}
\hline Symbolic form & Template & Schema & QM & E\&M \\
\hline "Same amount" form & $\square=\square$ & Two amounts are the same & $8(\mathrm{~S} 1-\mathrm{S} 8)$ & 8 (S2, S4, S9-S14) \\
\hline \multirow[t]{2}{*}{$\begin{array}{l}\text { "Parts-of-a-whole" } \\
\text { form }\end{array}$} & $\square+\square+\cdots$ & $\begin{array}{l}\text { Amounts of substance } \\
\text { sum to whole }\end{array}$ & $\begin{array}{l}\text { Situation } 1: \mathrm{P} \text { arts of } 5(\mathrm{~S} 1, \mathrm{~S} 3, \mathrm{~S} 4, \mathrm{~S} 6) \\
\text { the region }\end{array}$ & $4(\mathrm{~S} 4, \mathrm{~S} 10, \mathrm{~S} 12, \mathrm{~S} 13)$ \\
\hline & & & $\begin{array}{l}\text { Situation 2: P arts of } 3(\mathrm{~S} 2, \mathrm{~S} 5, \mathrm{~S} 7, \mathrm{~S} 8) \\
\text { the source }\end{array}$ & $\begin{array}{l}5(\mathrm{~S} 2, \mathrm{~S} 9, \mathrm{~S} 11 \\
\mathrm{S} 12, \mathrm{~S} 13, \mathrm{~S} 14)\end{array}$ \\
\hline "Whole-part" form & $\square-\square$ & $\begin{array}{l}\text { A new amount from taking } \\
\text { away a piece of a whole }\end{array}$ & 0 & $1(\mathrm{~S} 9)$ \\
\hline "Opposition" form & $\square-\square$ & $\begin{array}{l}\text { Two terms from } \\
\text { opposing influences }\end{array}$ & $1(\mathrm{~S} 2)$ & 0 \\
\hline
\end{tabular}

hitting here. If it is transmitted, then it is going to be continuous. In a sense that whatever come through this side, has to equal something like that. If it is transmitted, then it is going to be continuous. In a sense that whatever come through this side, has to equal something like that (writing down $\square=\square$ )." (See Fig. 4.)

Here we can see this student drew a template structure of the equation explicitly $(\square=\square)$ without any prompts from the interviewer, suggesting that without filling in the blanks they were using the "same amount" symbolic form when constructing equations. The conceptual schema the student used when constructing this equation is that the incoming wave has to equal to something on the other side (the transmitted) or something has to be continuous at the barrier. They expressed an intuitive idea about the structure of the equation but at this stage they did not show exactly what physics quantity they are equating to one another. For example, in this interview, after the interviewer further prompted the students:

I: "So when you have this equals this. On the two sides of the equation, what are the two side of this equation?" S3: "What I am trying to show is sort of not quantitative in the sense. The left side is the incoming has to equal the transmitted part. No, because you have the reflected part too. Because as I recall, say like an infinite square well. Yeah, I am confused. I kind of lost there."

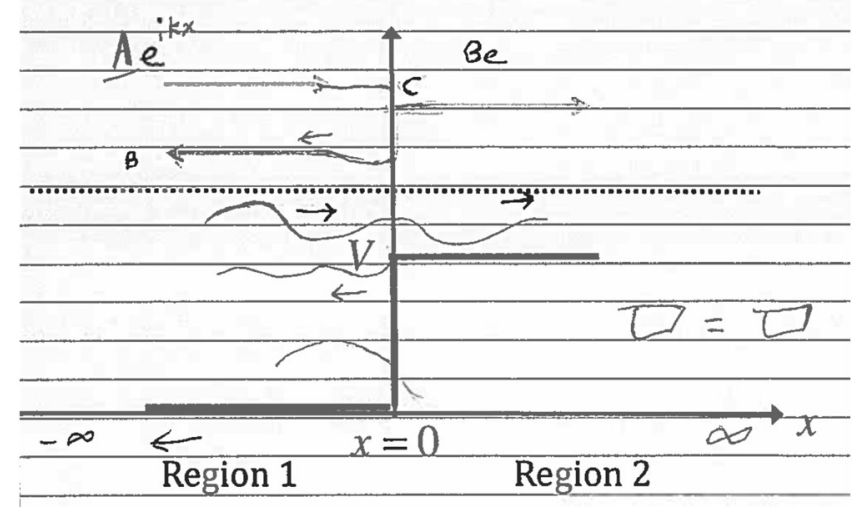

FIG. 4. Student's explicit drawing of symbolic template (QM, S3).
We can see that students can have the general correct structure for building an equation; the difficulty seems to lie in the conceptual underpinnings of the equation: what physical quantities are they equating to each other. After getting stuck, S3 was prompted by the interviewer using the scaffolding question. The student then continued on with the construction of the expression.

Below we gave another example of the E\&M question where students were thinking more explicitly about the physical quantities.

S10: "We have two linear media. We can use the same equation we had before of $E_{1}^{\|}$equals $E_{2}^{\|}$. And $\epsilon_{1} E_{1}^{\perp}$ equals $\epsilon_{2} E_{2}^{\perp}$. Fortunately, we know $E_{1}^{\perp}$ is just 0 because it's not perpendicular to the boundary. So $E_{2}^{\perp}$ is also 0."

Here we see this student explicitly used boundary conditions of the parallel and perpendicular electric field ( $\epsilon_{1} E_{1}^{\perp}$ equals $\epsilon_{2} E_{2}^{\perp}$ is not correct though). The conceptual schema in this "same amount" form of their equation is the idea of the parallel component of the electric field being equal in two media.

\section{Parts of a whole}

The symbolic template for this form is $\square+\square+\cdots$. Sherin defined this symbolic form to be as follows: Amounts of generic substance, associated with terms, that contributes to a whole. The symbol template is two or more terms separated by a plus sign. With the use of the same "parts-ofa-whole" symbolic form, the way this symbolic form is contextualized can be different. We saw two different types of conceptual understandings students had using this form. Depending on students' focus in a given context, they invoked parts of a whole due to either a focus on the wave components in a given region or a focus on the wave source as the whole quantity. Both are equally common among students. Below we give examples of both situations.

Situation 1: Students' focus on the region.-For student's focusing on the region when constructing their equations, one side of the equation $(\square+\square=\square$ ) has two parts: the incoming and the reflected are "parts of" the "whole" 
region: region 1 . Here we provide two examples of students using parts of a whole with attention to the region, first for the QM question, and second for the E\&M question.

S3: "So we have $A e^{\left(-i k_{1} x\right)}+B e^{\left(i k_{1} x\right)}=C e^{\left(-i k_{2} x\right)}$. We have a wave coming from the left side. And then we are adding also the reflected wave. That equal the transmitted wave."

I: "why would the left-hand side of the equation consist of two terms?"

S: "I guess I look at everything on the left side, on the negative $x$-axis are grouped together versus everything on the right of the $x$-axis."

The reasoning the student provided for grouping the incoming and reflected waves together is that they are all parts associated with region 1 . That is the correct interpretation when applying boundary conditions, since the incoming and reflected wave are superimposed to form the wave in the region. Student responses were similar in the E\&M context.

S12: "The $E_{1}^{\perp}=E_{2}^{\perp}=0 \mathrm{cuz}$ the $E$ field is in the perpendicular direction to the boundary, uh, parallel to the boundary, not perpendicular to the boundary. So those are zero. The parallel components of $E$ is not zero, so we have $E_{1}^{\|}$equals $E_{2}^{\|}$. And $B_{1}^{\|} / \mu_{1}$ equals $B_{2}^{\|} / \mu_{2}$. In general, we have $E_{1}^{\|}$is the reflected $E$ field plus the incident $E$ field. And $E_{2}^{\|}$, that's in the same direction, so we have that."

In these cases, students' focus is on the region as they construct the "whole," subsequently summing the incoming and reflected waves as those are the parts that make up the "whole" wave in the region.

Situation 2: Students' focus on the source.-For students focusing on the source when constructing their equation, one side of the equation $(\square=\square+\square)$ has two parts: the reflected and the transmitted are "parts of" the "whole" source: the incoming wave (serving as the source of the whole). Again we provide two examples of students' reasoning focusing on the source, first in the E\&M context and then from QM. Note that this naive reasoning can be connected to correct physics principles. In QM, the reflection and transmission coefficient (not wave function itself) does add up to be 1. In E\&M, the total energy (not the $E$ field itself) of the incoming waves does equal to the sum of the reflected and transmitted waves. However, as demonstrated below, students were not relating the accurate concepts with the particular symbolic template they chose.

S11: "Everything has to add up here. Everything that comes out has to add up to what comes in."
I: "You are saying something has to add up?"

S: "Well you can't just like come up with more wave than you had going in. So the magnitude of this coming in has to add up, I suppose. Yes, because some of it will go through and some of it will go back. We can't just create more electric field than you had coming in, I think."

I: "You said this equals something?"

$S$ : "Yes, so the magnitude of the reflected wave expression at the boundary plus the magnitude of the transmitted waves expression should equal to the magnitude of the incident waves."

Students had this underlying conceptual understanding: The incoming splits into reflected and transmitted, and therefore the incoming should equal the reflected plus transmitted. Below we show another example from student working on the QM question; the idea in situation 2 is much more subtle. In fact, students initially wrote down $A+B=C$, which is the correct relationship. But later on, while explaining their reasoning we can see they had an incorrect conceptual understanding of the equation.

S2: "This is at $x=0$. Because this is like one thing (referring to the reflected), and that is the other (referring to the transmitted). So instead of forgetting that... So it's $A+B=C$. I mean that's the obviously one. [after a while] it kinds of make sense because first it is this one. And then when it comes here, it sort of get split into this one and this one. Now since this one is going in the opposite direction. That's the negative. So this will be this equal this one plus this but it's negative [referring to $A=-B+C$ without writing it down]. So this equal this minus this. And I just like move it over here so $A+B=C$."

We can see from the above example, even though the student wrote down a correct final expression $A+B=C$, they were not constructing this equation themselves; one might speculate they were writing it down simply from memory. Then the student tries to make sense of the answer, using the conceptual understanding where the incoming splits into reflected and transmitted. However, this student added a negative sign for the reflected term and obtained a correct result by accident.

Overall, we find that this underlying conceptual understanding about the incoming splits into reflected and transmitted is common among both students working through E\&M and QM contexts. The general idea is not wrong. In QM, the reflection and transmission probability (not the wave function itself) add up to be 1. In E\&M, the total energy (not the $E$ field itself) of the incoming waves equal to the sum of the reflected and transmitted waves. However, students were not relating the accurate concepts with the particular symbolic template they chose. 
Mixed focus on the region and the wave source.-In one case we see the student attended to both the region and the wave source at different stages of their reasoning process.

S13: "The one and two refer to the mediums, so I know that the incident wave is gonna pass through this boundary and into medium 2, so all I do is to replace the incident with the trans, oh, I replace the incident with the one, replace the two with trans(mitted)."

I: "What about the reflected?"

S: "Oh, that's a good point [pause]... Because going from one medium to another, I'm not going to another medium, this is the same medium. The incident and the reflected are in the same medium, make sense to me that they are the same, but that means doubling the amplitude."

I: "What do you mean by 'doubling'?"

S: "That means when I'm seeing one beam of light being transmitted, and then reflected, out of the same strength as both of the transmitted and the reflected beams. so that wouldn't make sense. But I don't know how to fix that. It seems to be some percentages, both of these added up together should give me that, that's what it should be, there should be a plus sign. Oh, that's how you should do it. Because, wait... [pause]. The electric field in one medium is equal to the electric field in another medium. But that doesn 't make sense to me. Kinda make sense to me that this would be over here, just because, if I have an incident amplitude of this, it's gonna split into reflected and transmitted wave. Actually, it makes sense to me that this would be equal sign, and this would be the plus sign. intuitively that makes sense." (See Fig. 5.)

This student wrote down boundary conditions and said the electric field in medium 1 is equal to the electric field in medium 2. In the very beginning, the student said they would replace the incident with 1 (medium 1) and the transmitted with 2 (medium 2); they had the right idea except simply forgetting the reflected part. The interviewer reminded the student about the reflected wave. However, the student thought by combining the incoming and reflected wave together, they would be doubling the "strength." Eventually the student opted for a focus on the wave as the "whole," where they combined the reflected and the transmitted term together (Fig. 5).

\section{Whole-part}

The symbolic template for this form is $\square-\square$. The definition for "whole-part" is a new net amount is produced by taking away a piece of an original whole. Previously we discussed that different conceptual ideas (region and wave source) can be associated with the same symbolic form (parts of a whole). Here we see that the reverse can also happen where the same conceptual idea could also be associated with different symbolic forms. Although we only show the possibility of such an occurrence rather than

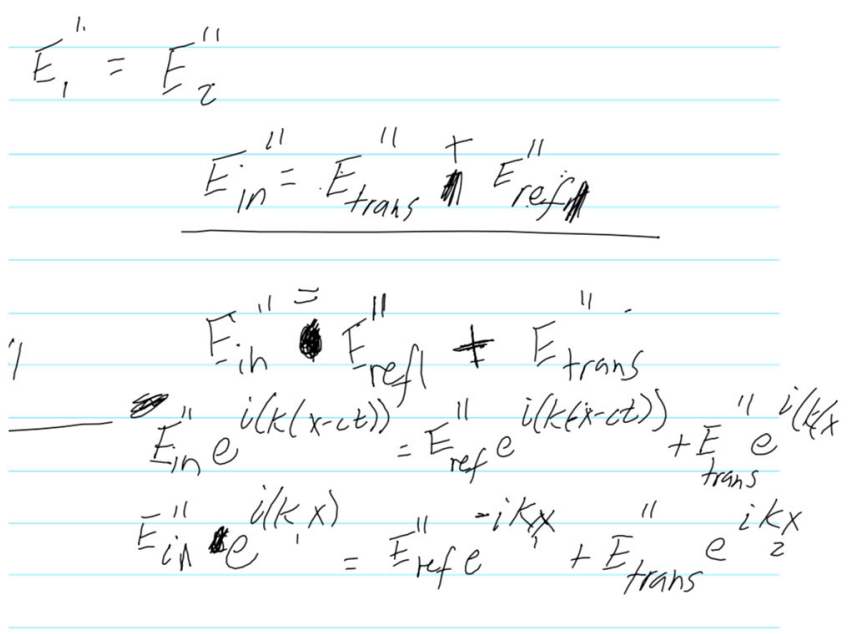

FIG. 5. Students' work (E\&M, S13).

claiming this as a trend given that we only observed this with one student (S9, E\&M). This student who had the same "energy/probability conservation" idea could write down a different symbolic template $(\square-\square)$, viewing the transmitted being a new amount produced by taking away the reflected from the incoming.

S9: "I can't remember clearly. But the incident wave has a probability of 1 . And probability transmitted of and probability of reflected need to be equal to 1 . $1=\operatorname{Prob}(T)+\operatorname{Prob}(R)$. So, it is actually $E_{I}-E_{R}=$ $E_{T}$. So, $1-\operatorname{Prob}(R)=\operatorname{Prob}(T)$. I think it is related to, you can take the incident wave and say what portion [... inaudible] and what happen to the other two? So I think it is a subtraction because if you take away the amount of reflected, you should have the amount of transmitted."

There was a mix of different elements in students' reasoning. When they referred to the probability of the transmitted and reflected waves, they were using concepts from quantum mechanics while the question at hand involves electromagnetic waves. But the reasoning of these two probabilities has to add to one is correct. In the context of electromagnetic waves, the analogous equation should be the reflection coefficient and transmission coefficient add up to one; or one could say the intensity of the incident wave is equal to the intensity of the reflected wave plus the transmitted. The student used electric field to be the physics quantity in that equality, confusing the electric field with either the intensity of the wave or the reflection-totransmission coefficient.

\section{Opposition}

The symbolic template for this form is $\square-\square$. Two terms, separated by a minus sign, associated with influences that work against each other. Opposition was 
given in Sherin's paper as a separate symbolic form, and it differs from parts of a whole in that it emphasizes the opposite influences. Despite having the same symbolic template, it also differs from whole-part because the minus sign comes from opposite influences rather than taking away a portion of a whole amount.

Opposition refers to a situation in which the two influences compete in a specific manner: they are opposing influences. This conceptual opposition is associated with the use of a negative sign in the equation. We see an example where students use the "opposition" symbolic form, although it is not entirely parallel with the use of other symbolic forms. Below we give the example where the student talks about the reflected wave going the opposite direction and therefore there should be a minus sign in front of the wave function, but the use of opposition is nested within the parts-of-a-whole form.

S2: "And then when it comes here, it sort of get split into this one and this one. Now since this one is going in the opposite direction. That's [B] the negative. So this will be this equal this one plus this but it's negative. So this equal this minus this. And I just like move it over here so $A+B=C$."

As mentioned previously, this student (S2) first used the parts-of-a-whole symbolic form, attending to the incoming wave as the whole, and wrote down $A=B+C$, then they used the opposition form and added a minus sign in front of $B$, resulting in the final expression of $A+B=C$. The use of opposition symbolic form here is subtle and it is hard to separate it entirely from the use of parts of a whole, but the underlying conceptual understanding the student had was that the reflected wave is in the opposite direction and therefore gives an opposite influence from the incoming and transmitted ones.

\section{Other possible forms}

This paper only focuses on the overarching symbolic forms students used while constructing equations of boundary conditions without discussing the sublevel structures. Depending on the complexity level of the question, sublayer symbolic forms can exist. Each symbolic template could contain another layer of a symbolic template. For example, within each $\square$, some students wrote down a constant $(A)$, and some wrote down a constant times some other function $\left(A e^{-i k x_{\ldots}}\right)$. Sometimes students also used derivatives $(d / d x)$ when constructing boundary conditions. Potential new symbolic forms need to be identified in order to better describe these fine-scale activities. Even though these forms look like the "dying-away" symbolic form that was identified in Sherin's work earlier (a whole dies away with some parameter that appears raised to a negative power in the exponent of an exponential) on the surface, we cannot conclude these forms were used in this context without further analysis. Without further analysis, we cannot conclude these forms were used in this context. Questions used in this analysis are broad enough that the overarching structures appear without dwelling too much on the details. A different kind of question is needed to better isolate the kind of symbolic forms of interest and probe fine-grained details.

\section{ALTERNATIVE MODEL}

It is important to note that using symbolic forms is not the only way to explain student reasoning. First, we review and summarize Sherin's discussion. One of the problems provided to students in Sherin's paper has to do with air resistance. Imagine two objects are dropped from a great height. These two objects are identical in size and shape, but one object has twice the mass of the other object. Because of air resistance, both objects eventually reach terminal velocity. The question asks students to compare the terminal velocities of the two objects. It also asks whether the answer differs if there was a wind blowing straight up when the objects were dropped and what if the wind was blowing straight down. Students Mike and Karl composed the following expression: $a(t)=-g+[f(v)] / m$. Mike says, "So, at least we can agree on this and we can start our problem from this scenario [e.g., indicates the diagram with a sweeping gesture]. Right? Okay? So, at any time, at any time, the acceleration due to gravity is G, and the acceleration due to the resistance force is $\mathrm{F}$ of $\mathrm{V}$ over M [writes $g+f(v) / m$ ]. This is mass $\mathrm{M}$ [writes $m$ next to the mass on the diagram]." Karl says, "Ah." Mike says, "Okay, now they're opposing so it's a minus." One of the symbolic forms Sherin used to describe students' thinking is opposition. Opposition refers to a situation in which the two influences compete in a specific manner: they are opposing influences. This conceptual opposition is associated with the use of a negative sign in the equation ("Now they're opposing so it's minus"). Sherin said these students understood the situation in terms of two competing influences, and they directly embodied this understanding in an equation.

Sherin also acknowledged that symbolic forms are not the whole story to equation construction and more formal considerations and remembered formulas must also play a role. Another alternative model was the perspective of principle-based schemata view which was worked out by Larkin [17]. According to Larkin, experts possess a number of schemata that are directly associated with physical principles. For example, Larkin wrote that the forces schema corresponds to the physical principle that the total force on a system (along a particular direction) is equal to the system's mass times its acceleration. This is essentially the physics principle of Newton's second law.

One crucial difference between Larkin's view and the symbolic form view is that the former is closely tied to physical principles. For Larkin, it is crucial that the influences 
in competition are forces because Newton's laws only involve forces. In contrast, in the view of symbolic forms, the situation is generically schematized as competing influences - a notion that cuts across physical laws and just refers to the mathematical justification. Sherin's analysis suggests that in a typical problem-solving episode, a qualitative conceptual account was partly driving the students' work with equations, with symbolic forms mediating the interaction between conceptual understanding and symbolic expressions. It is worth noting that these two models are not two competing perspectives: the use of symbolic form perspective does not deny the existence of the other model.

Authors of this paper concur with Sherin that in some cases the use of symbolic form sometimes explains students' reasoning better. In the context of constructing equations of boundary conditions, we repetitively find that students have an intuitive understanding that drives the structures of the equations they wrote. For example, they have the idea of same amount, that something has to equal to something else. This generic intuition and sense students had drives them to write down the equation. The physics principle is boundary conditions, but we observe that oftentimes students did not start with this physics principle. Students' use of symbolic forms to construct equations is still very common at the upper-division level as shown by our study.

The use of symbolic form perspective also did not deny the existence of students' use of principle-based schemata. We do observe that some students were indeed starting out from the physics principles of boundary conditions of $E 1=E 2$. In fact, situation 1 associated with the parts-ofa-whole symbolic form can also be explained using the principle-based schemata. The reason that we chose the perspective of symbolic form is the following: first, not all students wrote out $E 1=E 2$ explicitly, but they did express the idea of associating the incoming and reflected wave together because they all belong to the same region. Their conceptualization of the situation took shape as an equation. Another reason we use the symbolic form perspective is that situation 2, which was used by students just as frequently as situation 1, was not based on any explicit physics principle. The idea of two parts (reflected plus transmitted) make up the whole (incoming) thing is a general conceptual idea, rather than a particular physics principle. If one starts from the physics principle of conservation of energy, then only energy (intensities of the wave) should be used. However, students have this general idea of parts of a whole - a notion that cuts across physics laws - many types of entities, other than energies, can add up to a whole amount. We believe students' work using situation 2 is better explained by symbolic forms. The differences between students who used situation 1 (also oftentimes answer it correctly) and students who used situation 2 (tend to get it wrong) are not that drastically different. The difference of the two situations is which two parts count toward the "whole" which connects to the physics concepts. Students' differences in this conceptual understanding drive them to write down different equations. Therefore, we believe it is productive to use symbolic forms because it helps narrow down the conceptual underpinnings of equation construction and can offer possible pedagogical implications (discussed further in Sec. VI).

\section{CONCLUSIONS AND DISCUSSIONS}

This study uses symbolic form as the theoretical framework to investigate how students construct equations for boundary conditions in two different contexts: potential step in quantum mechanics and electromagnetic waves across media in electrodynamics. We adapted typical textbook problems and conducted one-on-one think-aloud interviews with students. The symbolic form analysis enables us to see what concepts students use to construct equations. It also shows that we need to identify new symbolic forms in the upper-division contexts.

As a result of this study, some symbolic forms previously identified in Sherin's work for introductory physics were also identified in upper-division students' work: same amount, parts of a whole, whole-part, and opposition. Further work is needed in order to identify nested sublayer symbolic forms or other new forms. Generally speaking, students were able to recognize the general structure needed for the equation and generate correct symbolic templates. However, even when students possess many of the necessary symbolic forms, they still struggle with the details of what goes into the specific symbolic templates, as shown in other work with symbolic forms [11]. As an instructor, helping students to get these elements organized in the right place is not a trivial goal [5].

There are a couple of implications for instruction. First, as mentioned, we found that students possess many resources needed to understand equations and often do obtain the correct general structures of boundary conditions. As instructors, we would like to get these resources engaged more frequently and at the right times. For example, students tend to know something has to be continuous and something has to equal to something else. When they could recall the exact format of a physics principle from memory, they tended to follow that memory. But when they cannot recall the exact format of such a physics principle, they tend to use a more naive conceptual understanding to construct an equation (which is not a bad thing). One possible first step in instruction could be asking students to think about what exactly is continuous when going over physics principles, and hopefully by emphasizing the conceptual underpinnings of these equations, students could strengthen their own conceptualization with the structure of these equations. Of course many instructors may already do this, but a little more frequency and emphasis could help.

Other than utilizing the strength and resources students already have, instructors could also direct emphasis to 
particular areas for these types of problems. For example, we found similar patterns in the use of these symbolic forms between the QM and E\&M contexts. Instructors could point out the similarities on purpose between contexts and help students see the similarities between the underlying structures of the equations. Doing this explicitly could also help students avoid confusion when they switch from one context to another. Furthermore, we found different conceptual ideas that students associated with the same symbolic form; e.g., in the part-of-a-whole form, students consider that the two terms are either parts of the "same region" or parts of the "same incoming source." This suggests that instructors could pay more attention to emphasizing the conceptual underpinnings of boundary conditions; i.e., what are we setting equal to each other at two sides of the equation? In addition to writing and manipulating equations, instructors could ask students what those equations mean. They can even compare the two different conceptual models when setting up boundary conditions.

Analyzing students' reasoning in upper-division courses is complicated. There are several limitations to this study. First, one-on-one interviews were conducted because of the exploratory nature of the study. While the interviewer remained as objective as possible, the process was still of a laboratory nature rather than authentic. Second, some drawbacks can occur due to the choice of these specific questions. Both interview questions are common scenarios that students may have encountered several times in class. We chose these scenarios because of their shared similarity across two physics contexts (QM and E\&M). The drawback is the difficulty in distinguishing whether students construct equations simply by recalling from memory. These questions are also very specific in the way they asked for the students' answer. For example, the questions asked students to write a mathematical expression that relates the amplitudes of the waves with the intention of better isolating the area of interest. It is yet to be discovered whether similar student reasoning would occur if the questions asked students to calculate the reflection coefficient which is more typical for a homework problem students see but also requires an even longer process with an even less clear focus. Last, both interview questions still require a multistep process while a more fine-grained question could have the advantage if we want to investigate a particular aspect of symbolic forms in detail. For example, current questions do not focus on the sublayer of symbolic forms.

Future work includes running interviews with a group of students rather than a one-on-one interview to obtain a more authentic process. One could also craft novel scenarios and unconventional questions to address the concern where students simply recall the information. In addition, different types of questions could be asked to see whether they impact how students use symbolic forms. For example, we could ask students to calculate the reflection coefficient instead of the relationship between amplitudes of the waves. Lastly, a more fine-grained question can allow for a detailed analysis such as identifying new symbolic forms when constructing complex exponential expressions.

\section{ACKNOWLEDGMENTS}

We gratefully acknowledge Andrew Elby for his original suggestion of applying the symbolic form framework and Eleanor Sayre for her insightful comments. We greatly appreciate Homeyra Sadaghiani for careful review and critique of the paper. We also thank Steven Pollock for his insight and conversation. Special thanks to Thu Chau for her diligent work on transcribing interviews. Part of the data used in this study were collected under the support of NSF-CCLI Grant No. 1023208.
[1] D. E. Meltzer and R. K. Thornton, Resource letter ALIP-1: Active-learning instruction in physics, Am. J. Phys. 80, 478 (2012).

[2] B. Wilcox, M. Caballero, D. Rehn, and S. Pollock, Analytic framework for students' use of mathematics in upper-division physics, Phys. Rev. ST Phys. Educ. Res. 9, 020119 (2013).

[3] R. Pepper, S. Chasteen, S. Pollock, and K. Perkins, Observations on student difficulties with mathematics in upper-division electricity and magnetism, Phys. Rev. ST Phys. Educ. Res. 8, 010111 (2012).

[4] R. Pepper, S. Chasteen, S. Pollock, and K. Perkins, Facilitating faculty conversations: development of consensus learning goals, Proceedings of the Physics Education Research Conference 2011, Omaha, Nebraska (2011),
https://www.compadre.org/Repository/document/ServeFile .cfm?ID=11870\&DocID=2718.

[5] B. Sherin, How students understand physics equations, Cognit. Instr. 19, 479 (2001).

[6] A. A. diSessa, Knowledge in pieces, in Constructivism in the Computer Age, edited by G. Forman and P. B. Pufall, The Jean Piaget Symposium series (Lawrence Erlbaum Associates, Inc., Hillsdale, NJ, 1988), pp. 49-70.

[7] D. Hammer, Student resources for learning introductory physics, Am. J. Phys. 68, S52 (2000).

[8] C. A. Manogue, P. J. Siemens, J. Tate, K. Browne, M. L. Niess, and A.J. Wolfer, Paradigms in Physics: A new upper-division curriculum, Am. J. Phys. 69, 978 (2001).

[9] D. Meredith and K. Marrongelle, How students use mathematical resources in an electrostatics context, Am. J. Phys. 76, 570 (2008). 
[10] E. Kuo, M. M. Hull, A. Gupta, and A. Elby, How students blend conceptual and formal mathematical reasoning in solving physics problems, Sci. Educ. 97, 32 (2013).

[11] B. P. Schermerhorn and J. R. Thompson, Physics students' construction of differential length vectors in an unconventional spherical coordinate system, Phys. Rev. Phys. Educ. Res. 15, 010111 (2019).

[12] B. W. Dreyfus, A. Elby, A. Gupta, and E. R. Sohr, Mathematical sense-making in quantum mechanics: An initial peek, Phys. Rev. Phys. Educ. Res. 13, 020141 (2017).
[13] S. Jones, Understanding the integral: Students' symbolic forms, J. Math. Behav. 32, 122 (2013).

[14] B. Schermerhorn, Ph.D. thesis, University of Maine, 2018.

[15] N. Becker and M. Towns, Students' understanding of mathematical expressions in physical chemistry contexts: An analysis using Sherin's symbolic forms, Chem. Educ. Res. Pract. 13, 209 (2012).

[16] S. Mayer, The early evolution of Jean Piaget's clinical method, Hist. Psychol. 8, 362 (2005).

[17] J. Larkin, in Mental Models, edited by D. Gentner and A. Stevens (Lawrence Erlbaum, Hillsdale, NJ, 1983), p. 75. 